\title{
AVALIAÇÃO DA QUALIDADE DE VIDA SUBJETIVA DOS IDOSOS: uma comparação entre os residentes em cidades rurais e urbanas
}

\author{
Cíntia Ribeiro Martins ${ }^{1}$ \\ Francisco José Batista de Albuquerque \\ Charlene Nayana Nunes Alves Gouveia ${ }^{3}$ \\ Carla Fernanda Ferreira Rodrigues ${ }^{4}$ \\ Maria Tereza de Souza Neves 5
}

\section{Resumo}

Este estudo objetivou avaliar a Qualidade de Vida Subjetiva (QVS) de idosos residentes nos meios urbano e rural do Estado da Paraíba. Participaram 284 idosos, 56\% moradores do ambiente urbano e $44 \%$ moradores do ambiente rural. Foram utilizados, como instrumentos, a escala WHOQOL-bref e um Questionário Biodemográfico para a caracterização da amostra. Os resultados sugerem que, de modo geral, os idosos possuem um índice de QVS satisfatório ( $\mathrm{M}=3,47$; $\mathrm{DP}=0,43)$. Comparando-se os grupos em função do gênero e do ambiente em que vivem, não há diferença significativa nos índices de QVS, porém estes índices correlacionam-se direta e significativamente com o nível econômico. Pode-se concluir que as condições de vida dos meios urbano e rural, apesar das diferenças, não influenciam na QVS dos idosos. O que faz reduzir a satisfação com suas condições de vida é a falta de recursos financeiros, sendo os grupos de menor poder aquisitivo dos que demonstraram os menores índices de QVS.

Palavras-chave: Qualidade de vida. Idosos. Ambientes rural e urbano.

\footnotetext{
${ }^{1}$ Mestranda do Programa de Pós-graduação em Psicologia Social da Universidade Federal da Paraíba - (UFPB) e Bolsista da Coordenação de Aperfeiçoamento de Pessoal de Nível superior (CAPES). E-mail: cintiarmartins@uol.com.br

2 Professor do Departamento de Psicologia e do Programa de Pós-Graduação em Psicologia Social da UFPB. E-mail: francisco.albuquerque@pesquisador.cnpq.br-frajoba@uol.com.br

${ }^{3}$ Aluna do Curso de Graduação em Psicologia da UFPB e Bolsista do Programa Institucional de Bolsas de Iniciação Científica (PIBIC/UFPB/CNPq). E-mail: nayanagouveia@yahoo.com.br

${ }^{4}$ Aluna do Curso de Graduação em Psicologia da UFPB e Bolsista do Programa Institucional de Bolsas de Iniciação Científica (PIBIC/UFPB/CNPq). E-mail: carlafernandafr@gmail.com

${ }^{5}$ Mestranda do Programa de Pós-graduação em Psicologia Social da UFPB. E-mail: terezapsi@hotmail.com

Estud. interdiscip. envelhec., Porto Alegre, v. 11, p. 135-154, 2007.
} 


\section{Introdução}

O aumento da longevidade e o crescimento do número de idosos, como nunca antes foi visto na história da humanidade, aliados às políticas de proteção social aos idosos, podem responder pela mudança de uma perspectiva pessimista da velhice, que passou a ser vista de modo mais positivo, como uma experiência mais gratificante. Neste sentido, muitos estudos têm se dedicado a estudar a velhice sob novas óticas, como qualidade de vida, bem-estar subjetivo, contentamento, esperança e satisfação com a vida, entre outros. A maioria desses estudos se refere a idosos que habitam em ambientes urbanos, sendo poucas as pesquisas que abordam o tema do envelhecimento no ambiente rural. Caracterizam o ambiente rural os municípios que possuem menos de 25.000 habitantes. Aproximadamente $75 \%$ dos municípios brasileiros se enquadram nesta definição e neles residem cerca de 40 milhões de pessoas. Estes municípios rurais são constituídos por um núcleo urbano, que corresponde à sede municipal, e um núcleo agrário, onde se desenvolvem atividades de agricultura e pecuária. Neste estudo, buscouse avaliar a qualidade de vida dos idosos, comparando os índices dos que residem no ambiente rural urbano com os que moram no ambiente urbano da Paraíba. Considerou-se como zona urbana a cidade de João Pessoa, capital da Paraíba, que possui aproximadamente 600 mil habitantes, de acordo com o IBGE (2000).

\section{Qualidade de Vida}

A preocupação com o tema da qualidade de vida emergiu a partir da Segunda Grande Guerra Mundial, quando foi usado o conceito de "boa vida" para referir-se à conquista de bens materiais. Em seguida, o conceito foi ampliado e passou a medir o quanto uma sociedade havia se desenvolvido economicamente. A criação de indicadores econômicos permitia comparar a qualidade de vida entre diferentes países e culturas. Depois, o termo passou a designar, além do crescimento econômico, o desenvolvimento social (PASCHOAL, 2000).

A partir da década de 60, percebeu-se que, embora os indicadores sócio-econômicos como a Qualidade de Vida Objetiva (QVO) fossem importantes, era necessário avaliar a qualidade de vida percebida 
pela pessoa, o quanto elas estavam ou não satisfeitas com a qualidade de suas vidas, ou seja, a Qualidade de Vida Subjetiva (QVS) (NERI, 2004; PASCHOAL, 2000). O indivíduo deveria julgar a qualidade de sua vida, e não apenas avaliar como se enquadrava ou não no modelo de qualidade de vida que o pesquisador julgava ser boa.

Várias disciplinas, com pretensões científicas, têm manifestado interesse e se debruçado sobre o conceito de qualidade de vida. Dentre elas, podem-se destacar a economia, a Psicologia, a Pedagogia, a Medicina e a Enfermagem, além da própria Filosofia. Cada uma delas parte de diferentes perspectivas e agrega diversos significados associados ao conceito de qualidade de vida, o que contribui para a falta de um consenso, entre os pesquisadores, sobre a sua operacionalização. Porém, apesar das diferentes abordagens, existe uma preocupação fundamental com os aspectos que contribuem para o bem-estar do homem, para um bom nível de saúde e de desenvolvimento humano.

Segundo Neri (2000), os motivos que levam à dificuldade de operacionalização da qualidade de vida são os seguintes: (1) o construto possui múltiplas dimensões; (2) resulta da atuação de muitos eventos concorrentes, isto é, é multideterminado; (3) refere-se à adaptação de indivíduos e grupos de pessoas em diferentes épocas da vida de uma ou várias sociedades; (4) é um evento dependente do tempo.

No início da década de 1990, o Grupo de Qualidade de Vida da Organização Mundial de Saúde (Grupo WHOQOL), equipe de especialistas reunida pela OMS para desenvolver um instrumento com a finalidade de medir o referido construto, definiu a qualidade de vida como "a percepção do indivíduo de sua posição na vida, no contexto da cultura e sistema de valores nos quais ele vive e em relação aos seus objetivos, expectativas, padrões e preocupações" (THE WHOQOL GROUP, $1994^{6}$ apud FLECK et al., 2000, p. 179). A multidimensionalidade desse construto deve abranger os seguintes domínios: 1 - físico; 2 - psicológico; 3 - nível de independência; 4 - relações sociais; 5 - meio ambiente; e 6 - aspectos espirituais (TRENTINI, 2004).

Nos estudos feitos, geralmente a qualidade de vida é composta por valores não-materiais, como amor, liberdade, solidariedade e in-

${ }^{6}$ THE WHOQOL GROUP. The development of the World Health Organization quality of life assessment instrument (the WHOQOL). In: Orley J, Kuyken W, editors. Quality of life assessment: international perspectives. Heidelberg: Springer Verlag; 1994. p. 41-60. apud FLECK, 2000, p. 179. 
serção social, realização pessoal e felicidade. Mas devem ser considerados também alguns componentes passíveis de mensuração e comparação, como a satisfação das necessidades mais elementares da vida humana, como alimentação, acesso à água potável, habitação, trabalho, educação, saúde e lazer, ou seja, objetos materiais que dão a idéia de bem-estar e conforto, assim como de realização individual e coletiva. O desemprego, a violência e a exclusão social podem ser reconhecidos como a negação da qualidade de vida, porém deve-se levar em conta a cultura de cada sociedade (MINAYO; HART; BUSS, 2000).

Nesse sentido, a qualidade de vida de um indivíduo ou de uma população apresenta aspectos objetivos e subjetivos (PASCHOAL, 2000), sendo ambos fundamentais para a sua avaliação, mensuração e compreensão.

Os entraves conceituais para definir a qualidade de vida dificultam também a elaboração de seus instrumentos de medida. Diante disso, ao se construir um instrumento deve-se procurar sintetizar sua complexidade, analisando as diferentes culturas e realidades sociais.

O Índice de Desenvolvimento Humano (IDH) foi um dos primeiros instrumentos criados para a mensuração da qualidade de vida, tendo sido elaborado pelo Programa das Nações Unidas para o Desenvolvimento (PNUD). O IDH foi criado com a finalidade de transpor o debate sobre desenvolvimento, de aspectos puramente econômicos - como nível de renda, produto interno bruto e nível de emprego - para aspectos de natureza social e também cultural. Neste indicador, considera-se que a renda, a educação e a longevidade são três elementos fundamentais da qualidade de vida de uma população e tem igual importância como expressão das capacidades humanas (MINAYO; HARTZ; BUSS, 2000).

$\mathrm{O}$ grupo de pesquisadores da OMS desenvolveu dois instrumentos para medir a qualidade de vida: o WHOQOL-100 e o WHOQOLBref. O primeiro instrumento é composto por 100 questões que avaliam seis domínios: físico, psicológico, nível de independência, relações sociais, meio ambiente e aspectos espirituais. $\mathrm{O}$ segundo instrumento corresponde a uma versão abreviada, com 26 questões, retiradas do anterior e cobre quatro domínios: 1 - físico, 2 - psicológico, 3 - relações sociais e 4 - meio ambiente (TRENTINI, 2004).

No presente estudo, para avaliar a qualidade de vida subjetiva 
preferiu-se utilizar o WHOQOL-Bref, devido à sua estrutura simplificada e compacta adequada a populações de baixa escolaridade e, muitas vezes, com restrições em suas capacidades cognitivas, como é o caso da amostra estudada. Este instrumento é composto por quatro domínios e engloba 02 questões gerais acerca da qualidade de vida e 24 subdomínios (facetas). O primeiro domínio é o físico e suas facetas são: dor e desconforto; energia e fadiga; sono e repouso; mobilidade; atividades da vida cotidiana; dependência de medicação ou de tratamentos; e capacidade de trabalho. O segundo é o psicológico, caracterizado pelos sentimentos positivos; pensar, aprender, memória e concentração; autoestima; imagem corporal e aparência; sentimentos negativos; e espiritualidade/religião/crenças pessoais. O terceiro refere-se às relações sociais e engloba as relações pessoais; suporte social; e atividade sexual. E o quarto domínio diz respeito ao meio ambiente, abrangendo a segurança física e proteção; ambiente no lar; recursos financeiros; cuidados com a saúde e sociais (disponibilidade e qualidade); oportunidades de adquirir novas informações e habilidades; participação e oportunidades de recreação/lazer; ambiente físico (poluição, ruídos, trânsito, clima); e transporte (FLECK et al., 2000).

A análise da qualidade de vida subjetiva torna-se um eficaz instrumento para conhecer a realidade social e como esta é percebida pelos integrantes de uma dada comunidade, devido à multidimensionalidade deste construto.

Através desta análise, foram avaliadas as percepções subjetivas dos idosos que residem em meio rural e dos que habitam em meio urbano, a fim de verificar as influências ambientais e culturais sobre suas aspirações e necessidades com relação à percepção da qualidade de suas vidas. A obtenção de dados de caracterização da qualidade de vida, do ponto de vista dos próprios idosos, é uma informação que pode ser fundamental para dinamizar medidas, através de políticas públicas adequadas a essa população, que permitam alcançar um envelhecimento bem sucedido.

O estudo da qualidade de vida em idosos é recente, mas, devido ao processo de envelhecimento populacional dos países desenvolvidos, a temática vem tomando evidência, principalmente porque há um fortalecimento da idéia de que velhice patológica demanda altos custos para o indivíduo e a sociedade (NERI, 2004). 
Com a chegada da velhice, a debilidade física, a dependência, a perda de papéis, os estereótipos e preconceitos, enfim, as inevitáveis perdas decorrentes do processo de envelhecimento, podem levar à diminuição da qualidade de vida dos idosos. Surge, então, a preocupação em transformar a sobrevida aumentada do ser humano numa etapa significativa da vida (PASCHOAL, 2000).

Para avaliar a qualidade de vida na velhice, é necessário adotar critérios de natureza biológica, psicológica e socioestrutural, devido à multiplicidade de elementos que exercem influência sobre o bem-estar do idoso: longevidade, saúde biológica, saúde mental, satisfação, controle cognitivo, competência social, produtividade, atividade, status social, renda, continuidade de papéis familiares e ocupacionais e continuidade de relações informais - principalmente rede de amigos (NERI, 1993; SANTOS et al., 2002). Alguns destes indicadores merecem destaque, por exercerem maior influência sobre a qualidade de vida do idoso, como a saúde, questões de dependência-autonomia e recursos financeiros.

Segundo Neri (1993), os recursos financeiros exercem um efeito compensatório sobre as perdas da velhice, o que denominou de "efeito tampão". As possibilidades de recursos ajudam no acesso à saúde e ao lazer, e na sensação de auto-eficácia, dando ao idoso a possibilidade de compensar perdas inevitáveis da velhice. Trentini (2004) observou, em seu estudo, que os idosos pertencentes aos níveis sociais mais baixos apresentavam piores índices de qualidade de vida em todos os domínios estudados, inclusive no tocante ao bem-estar subjetivo. A condição de aposentadoria e a falta de recursos econômicos também se mostraram significativamente associadas com uma pior qualidade de vida.

Mais uma vez, isto deve ser matizado. Dependendo do contexto social, o que aparentemente é pouco para uns, em ambiente de maior pobreza, pode fazer a diferença comparativa. Albuquerque, Lobo e Raymundo (1999) realizaram um estudo sobre os efeitos de benefícios sociais, como pensões e aposentadorias, recebidos por idosos em ambiente rural. Verificaram que, mesmo quando era equivalente apenas a um salário mínimo, o benefício tinha grande influência tanto na sua qualidade de vida, quanto na sua auto-estima. Por mais irrisório que seja, em uma condição de pobreza, este valor significa, muitas vezes, a única fonte de renda fixa de toda a família. Isto confere ao idoso um 
forte papel social perante seus familiares e a sua comunidade, pois agora pode ter inclusive crédito para comprar móveis e eletrodomésticos no comércio local, movimentando a economia do município.

Nesta pesquisa, foi analisada a relação entre o nível econômico e a QVS, assim como sua relação com o gênero. Os papéis sociais atribuídos ao homem e à mulher podem influenciar a percepção de sua QVS. Estudo realizado por Gouveia (2006), no ambiente rural da Paraíba, mostrou que os homens apresentavam-se significativamente mais satisfeitos com suas vidas do que as mulheres. Isto se deve, provavelmente, ao fato de que, ao atingirem a terceira idade, os homens idosos passam a conviver com a aposentadoria. Quanto às mulheres, por terem vivido a juventude em uma época em que não encontravam espaço no mercado de trabalho, tornam-se, em sua maioria, donas de casa e envelhecem cuidando de seus filhos e maridos. Nesta situação, só param quando já não têm mais condições físicas ou psicológicas para continuar desempenhando suas tarefas. Assim, enquanto os homens podem desfrutar sua velhice com mais tranqüilidade e maior descanso, livres das preocupações e do estresse, relacionados ao trabalho, as mulheres continuam executando suas funções domésticas. E, por não terem exercido outras atividades produtivas, muitas vezes se encontram excluídas dos benefícios sociais proporcionados àqueles que se enquadram nos padrões exigidos para a sua obtenção, e que são vinculados, quase sempre, ao exercício de atividades remuneradas.

Com esta pesquisa, deseja-se investigar se, em comparação com o meio urbano, o ambiente rural oferece condições mais vantajosas para os que envelhecem inseridos nesse contexto, proporcionando a percepção de melhor qualidade de vida. As condições diferenciadas que o meio rural oferece tornam singular a experiência de envelhecer nesse ambiente. Este fato requer a necessidade de adequação das políticas públicas direcionadas a essa parcela da população.

\section{Método}

Esta pesquisa foi desenvolvida através de um estudo correlacional, utilizando uma abordagem quantitativa. Foi avaliado o nível de qualidade de vida subjetiva (variável conseqüente) de idosos que vivem no meio rural e daqueles que vivem no meio urbano (variáveis antece- 
dentes), para realização de um estudo comparativo.

\subsection{Participantes}

Participaram deste estudo 284 idosos, dos quais 159 são moradores da cidade de João Pessoa, capital da Paraíba, e 125 vivem nos seguintes municípios rurais deste Estado: Rio Tinto, Itabaiana, Gurinhém, Mogeiro, Sobrado, Riachão do Poço, Pilar, Ingá, Salgado de São Felix, Mari e Juripiranga. Nos municípios rurais foram selecionados participantes que moram no núcleo urbano. Esta seleção foi realizada através de uma amostragem não-probabilística, por quota, considerando-se o ambiente (rural x urbano), o gênero (homem x mulher) e a renda familiar (alta, média e baixa). A idade média encontrada foi de 67,58 anos, com desvio padrão de 4,68, variando de 60 a 76 anos, apresentando-se aproximadamente igual nos diversos estratos, contando com $56 \%$ residindo em ambiente urbano e 44\% em ambiente rural, sendo 49,6\% homens e $50,4 \%$ mulheres. Em sua maioria, encontram-se casados $(65,1 \%)$, não concluíram o $1^{\circ}$ grau $(37,7 \%)$ e vivem em casa própria $(82 \%)$.

Com relação à renda familiar, na zona urbana $37,7 \%$ dos idosos apresentam renda familiar baixa, $39 \%$ renda média e $23,3 \%$ renda alta. No ambiente rural, a amostra foi composta por $48 \%$ de idosos de renda familiar baixa, $48 \%$ de renda média e $4 \%$ de renda alta. $\mathrm{O}$ baixo número de idosos com renda alta, no meio rural, ocorreu devido à dificuldade de encontrar pessoas com este nível econômico.

Foram considerados como idosos de baixa renda aqueles que possuem renda familiar de até 3 salários mínimos. Os idosos que possuem renda familiar entre 3 e 10 salários mínimos foram considerados de nível econômico médio. Aqueles que possuem renda familiar a partir de 10 salários mínimos foram considerados de nível econômico alto. $\mathrm{O}$ salário mínimo vigente no período da pesquisa (maio a junho de 2006) era de $\mathrm{R} \$ 350,00$.

\subsection{Instrumentos}

Foi utilizada a WHOQOL-bref - escala adaptada por Fleck et al. (2000) - para avaliar a qualidade de vida subjetiva. O WHOQOLbref consta de 26 questões, sendo duas questões gerais, sobre qualida- 
de de vida, enquanto as demais representam cada uma das 24 facetas que compõem o instrumento original. Foi realizada uma análise fatorial confirmatória para uma solução a quatro domínios (físico, psicológico, relações sociais e meio ambiente).

Utilizou-se, também, um Questionário Biodemográfico, que permitiu a caracterização da amostra quanto ao sexo, idade, estado civil, ocupação, escolaridade e formas de lazer, entre outros.

\subsection{Procedimento}

A coleta de dados foi realizada nas residências dos participantes, tanto na zona urbana como na zona rural, no período de maio a junho de 2006. Os pesquisadores, previamente treinados e orientados para intervir o mínimo possível nas respostas dadas pelos participantes, dirigiamse ao idoso, esclareciam o objetivo da pesquisa e solicitavam a sua colaboração. O aplicador explicava detalhadamente como o participante iria responder aos instrumentos, esclarecendo que deveriam escolher a resposta que melhor representasse o grau da sua satisfação com relação à situação mencionada em cada item pelo aplicador. Era enfatizada a ausência de respostas corretas ou incorretas. Em seguida, o aplicador marcava, no instrumento, a alternativa escolhida pelo idoso.

Durante todo o processo da pesquisa, foram obedecidos os princípios éticos utilizados em pesquisas com seres humanos estabelecidos na Portaria 196/96 do Ministério da Saúde (BRASIL, 1996). Esta pesquisa foi submetida e aprovada pela Comissão de Bioética do Centro de Ciências da Saúde - UFPB, sob o parecer de nº 1224.

\section{Resultados}

Com o objetivo de analisar o nível de qualidade de vida subjetiva dos idosos, utilizou-se a escala WHOQOL-bref, variando de 1 a 5 . Nesta escala, quanto mais se pontua próximo de 5 , melhor o nível de qualidade de vida subjetiva do idoso.

Para a realização de uma análise fatorial desta escala, foram utilizados, como indicadores, as medidas Kaiser Meyer Olkin (KMO) e o Teste de Esfericidade de Bartlett's (X2 $(\mathrm{gl})=$ Bartlett's). De acordo com essa análise, os resultados dos indicadores foram $\mathrm{KMO}=0,89$ e o 
$\mathrm{X} 2(325)=2425,36 ; \mathrm{p} \leq 0,00$. Com o objetivo de analisar a consistência interna da escala, foi utilizado o Alpha de Cronbach, que apresentou um índice de 0,84 .

De modo geral, os idosos obtiveram uma média de QVS igual a 3,47 , com desvio padrão de 0,43 . Através do teste t de student, verificou-se que, entre os idosos moradores do ambiente rural $(\mathrm{M}=3,46$; $\mathrm{DP}=0,40)$ e os idosos do meio urbano $(\mathrm{M}=3,48 ; \mathrm{DP}=0,45)$, não houve diferença estatisticamente significativa $(t=0,56 ; p>0,05)$ no índice de QVS, em função do ambiente em que vivem.

Os domínios que compõem a QVS foram analisados separadamente, a fim de verificar-se a influência do ambiente sobre cada um deles.

Tabela 1 - Comparação entre os domínios da qualidade de vida subjetiva em função do ambiente em que vivem.

\begin{tabular}{l|r|r|r|r|r|r|r}
\hline \multirow{2}{*}{ Domínios } & \multicolumn{3}{|c|}{ Média } & \multicolumn{3}{c|}{ Desvio Padrão } & \multirow{2}{*}{$\begin{array}{c}t \\
\text { (Rural } \boldsymbol{x} \\
\text { Urbano) }\end{array}$} \\
\cline { 2 - 7 } & Urbano & Rural & Geral & Urbano & Rural & Geral & \\
\hline Físico & 3,41 & 3,38 & 3,40 & 0,43 & 0,48 & 0,45 & 0,$41 ; \mathrm{p}>0,05$ \\
\hline Psicológico & 3,40 & 3,42 & 3,41 & 0,53 & 0,45 & 0,50 & $-0,30 ; \mathrm{p}>0,05$ \\
\hline Social & 3,84 & 3,91 & 3,87 & 0,61 & 0,63 & 0,62 & $-0,96 ; \mathrm{p}>0,05$ \\
\hline Meio Ambiente & 3,42 & 3,30 & 3,37 & 0,61 & 0,49 & 0,56 & 1,$74 ; \mathrm{p}>0,05$ \\
\hline
\end{tabular}

Conforme os resultados apresentados na Tabela 1, os índices nos domínios social e psicológico foram maiores no ambiente rural do que no ambiente urbano, enquanto nos domínios físico e meio ambiente, os idosos da zona urbana mostraram índices maiores, porém sem diferenças estatisticamente significativas.

Foram comparados os índices médios de QVS obtidos pelos homens idosos moradores do ambiente rural $(\mathrm{M}=3,43 ; \mathrm{DP}=0,42)$ com os índices dos homens idosos moradores do meio urbano $(\mathrm{M}=3,44$; $\mathrm{DP}=0,44)$, assim como se compararam os índices apresentados pelas mulheres idosas do ambiente rural $(\mathrm{M}=3,48 ; \mathrm{DP}=0,38)$ com os índices das mulheres idosas do meio urbano $(\mathrm{M}=3,53$; $\mathrm{DP}=0,45)$. Nos dois casos, não foram encontradas diferenças significativas $(\mathrm{t}=0,107$ e $\mathrm{t}=0,71$, respectivamente; $\mathrm{p}>0,05)$. 
De maneira geral, foram obtidos escores satisfatórios de qualidade de vida subjetiva, visto que estão mais próximos de cinco. Como os resultados indicam, não há diferença estatisticamente significativa nos índices de QVS em função do gênero, do ambiente, nem da interação destas variáveis. Ou seja, homens e mulheres, em ambiente rural ou urbano, apresentaram níveis de QVS estatisticamente semelhantes $(p>0,05)$.

Com o intuito de analisar a influência do nível econômico sobre este construto, a amostra foi estratificada em três níveis, de acordo com a renda familiar dos participantes. Para as análises comparativas entre estes níveis, utilizou-se como procedimento estatístico a ANOVA e, quando necessário, o teste de Comparação Múltipla através do Scheffe. Foram classificados como de renda baixa os idosos que possuíam renda familiar de até $\mathrm{R} \$ 1.050,00$, como de renda média os idosos que apresentaram renda familiar de R\$1.051,00 até R \$3.499,00, e como de renda alta os idosos que tinham renda a partir de $\mathrm{R} \$ 3.500,00$. A Tabela 2 permite visualizar as médias de renda familiar mensal, em cada nível de renda.

Tabela 2 - Renda familiar mensal média dos idosos em função do nível econômico

\begin{tabular}{l|r|r|r|r|r|r}
\hline \multirow{2}{*}{$\begin{array}{l}\text { Nível de } \\
\text { Renda }\end{array}$} & \multicolumn{3}{|c|}{$\begin{array}{c}\text { Média da Renda Familiar Men- } \\
\text { sal (R\$) }\end{array}$} & \multicolumn{3}{c}{ Desvio Padrão (R\$) } \\
\cline { 2 - 7 } & Urbano & \multicolumn{1}{|c|}{ Rural } & Geral & Urbano & \multicolumn{1}{c}{ Rural } & Geral \\
\hline Baixa & 637,67 & 628,25 & 632,96 & 235,99 & 191,51 & 214,05 \\
\hline Média & $1.791,55$ & $1.682,33$ & $1.737,84$ & 617,77 & 594,59 & 606,46 \\
\hline Alta & $6.716,22$ & $5.762,00$ & $6.602,62$ & $3.687,78$ & $1.436,39$ & $3.498,61$ \\
\hline
\end{tabular}

Para averiguar a influência do nível econômico sobre os índices de QVS, foram comparados os índices médios obtidos pelos idosos de renda baixa $(M=3,36 ; \mathrm{DP}=0,42)$, média $(\mathrm{M}=3,50 ; \mathrm{DP}=0,42)$ e alta $(\mathrm{M}=3,70 ; \mathrm{DP}=0,37)$. Através do teste estatístico ANOVA, foi encontrada diferença significativa entre os grupos $[\mathrm{F}(2,281)=10,97 ; p \leq 0,000]$. Para detalhar este resultado, realizou-se uma comparação múltipla através do Scheffe, conforme mostrado na Tabela 3: 
Tabela 3 - Comparação múltipla dos escores da Qualidade de Vida Subjetiva em função do nível de renda através do Scheffe

\begin{tabular}{c|c|r|r|r}
\hline $\begin{array}{c}\text { Nível } \\
\text { de Renda (I) }\end{array}$ & $\begin{array}{c}\text { Nível } \\
\text { de Renda (J) }\end{array}$ & $\begin{array}{c}\text { Diferença entre } \\
\text { as médias da } \\
\text { QVS (I-J) }\end{array}$ & $\begin{array}{c}\text { Desvio } \\
\text { Padrão }\end{array}$ & $\begin{array}{c}\text { Nível } \\
\text { Significância }\end{array}$ \\
\hline \multirow{3}{*}{ Baixa } & Média & $-0,13^{*}$ & 0,05 & 0,038 \\
\cline { 2 - 5 } & Alta & $-0,34^{*}$ & 0,07 & 0,000 \\
\hline \multirow{3}{*}{ Média } & Baixa & $0,13^{*}$ & 0,05 & 0,038 \\
\cline { 2 - 5 } & Alta & $-0,20^{*}$ & 0,07 & 0,024 \\
\hline \multirow{3}{*}{ Alta } & Baixa & $0,34^{*}$ & 0,07 & 0,000 \\
\cline { 2 - 5 } & Média & $0,20^{*}$ & 0,07 & 0,024 \\
\hline
\end{tabular}

* A diferença média é significante ao nível de 0,05.

Através desta análise, percebe-se que existe diferença significativa quando comparadas todas as combinações de níveis de renda $(p \leq 0,03)$. Os índices de qualidade de vida crescem de acordo com o nível econômico, ou seja, quanto maior a renda familiar, maior o índice de qualidade de vida subjetiva obtido pelo idoso.

A fim de verificar se os índices de qualidade de vida dos idosos diferem em função do local onde moram (rural ou urbano), sob a influência do nível econômico, foram comparados os escores médios de QVS dos idosos de renda baixa residentes na zona rural $(\mathrm{M}=3,39$; $\mathrm{DP}=0,43)$ com os da zona urbana $(\mathrm{M}=3,33$; $\mathrm{DP}=0,41)$, assim como se compararam os índices dos idosos de renda média que habitam no meio rural $(\mathrm{M}=3,51 ; \mathrm{DP}=0,37)$ com os que vivem no meio urbano $(\mathrm{M}=3,49$; $\mathrm{DP}=0,46)$. Apesar de existir uma pequena variação entre os escores dos idosos em função do local onde moram, tendo os idosos do meio rural, tanto os de nível baixo quanto médio, obtido maiores índices do que os idosos do meio urbano, esta diferença não foi considerada estatisticamente significativa $(p>0,05)$.

\section{Discussão}

Em uma escala variando de 1 a 5, na qual quanto mais perto de cinco maior a QVS, os idosos participantes deste estudo obtiveram índices satisfatórios, tanto no meio rural $(\mathrm{M}=3,46 ; \mathrm{DP}=0,40)$ como no 
meio urbano $(\mathrm{M}=3,48 ; \mathrm{DP}=0,45)$. Estes índices não mostraram diferença estatisticamente significativa em função do ambiente em que moram $(\mathrm{t}=0,56 ; p>0,05)$. Ou seja, independente do meio em que vivem, os idosos têm experimentado alto nível de QVS.

O alto nível de QVS, relatado pelos idosos, pode ser explicado pelo processo adaptativo dos indivíduos às condições da velhice. Segundo Stevens (2001) ${ }^{7}$ apud Sousa, Galante e Figueiredo (2003), a redefinição de seus objetivos, e a luta para alcançá-los, permite ao idoso ser um regulador da sua qualidade de vida, através do acúmulo de recursos que são úteis na adaptação à mudança. Ou seja, à medida que envelhecem e passam a lidar com as limitações impostas pela senilidade, os idosos entram em um processo adaptativo, através do qual passam a regular suas aspirações e a manter um equilíbrio entre perdas e potencialidades, conservando, assim, seu nível de QVS.

Para verificar a influência do ambiente sobre a QVS dos idosos, foram emparelhados os gêneros e comparados os índices das mulheres do meio rural com os das mulheres do meio urbano e os índices dos homens do ambiente rural com os dos homens do ambiente urbano. Constatou-se que em nenhum dos casos houve diferença estatisticamente significativa. Estes resultados contrariam o estudo de Sequeira e Silva (2002), realizado em Portugal, que concluiu que o ambiente rural oferece melhores condições para o envelhecimento do que o meio urbano. Provavelmente, as condições materiais daquele país, integrado ao Mercado Comum Europeu, nivelando as diferenças entre o ambiente urbano e o rural, permitem emergir os aspectos mais subjetivos da qualidade de vida dos seus idosos.

Além disso, os idosos do meio rural, atualmente, têm maior acesso tanto à aquisição de bens de consumo e aos meios de comunicação, devido às facilidades que o mercado oferece, quanto à energia elétrica, com a conseqüente popularização da televisão e do rádio, ampliando as suas oportunidade de diversão. $\mathrm{O}$ acesso a estes recursos diminui as diferenças antes existentes entre esses ambientes e permite aos idosos uma melhor qualidade de vida objetiva, o que, conseqüentemente, redunda em uma avaliação mais positiva da QVS.

Quando analisados separadamente, os domínios físicos ( $\mathrm{M}=3,40$;

STEVENS, N. Combating loneliness: a friendship enrichment programme for older women. Aging Soc 2001, v.21, p.183-202. apud SOUSA; GALANTE; FIGUEIREDO, 2003, p. 365. 
$\mathrm{DP}=0,45)$, psicológico $(\mathrm{M}=3,41 ; \mathrm{DP}=0,50)$, social $(\mathrm{M}=3,87 ; \mathrm{DP}=0,62)$ e meio ambiente $(\mathrm{M}=3,37 ; \mathrm{DP}=0,56)$ também apresentaram níveis satisfatórios, mantendo-se próximos de cinco. Ao se compararem os índices dos idosos moradores dos meios urbano e rural, em cada domínio, não foi verificada diferença estatisticamente significativa. Tratando-se do domínio físico, a ausência de variação justifica-se pelo fato dos idosos passarem por limitações e desconfortos físicos, seja qual for o lugar onde vivem, pois esta é uma condição que se reflete inteiramente nos seus próprios corpos. As causas até podem ser externas, mas são eles que apresentam a sintomatologia. Do mesmo modo, embora os aspectos psicológicos tenham relação direta com o ambiente, sua avaliação é de foro íntimo, com o julgamento sobre a vida e suas qualidades sendo inerente a cada pessoa. Por isto, os idosos residentes tanto em ambientes urbanos quanto em ambientes rurais refletem, neste domínio, um estado de espírito similar. Provavelmente, sua avaliação é balizada pelos que os rodeiam, o que acaba norteando seus sentimentos e julgamentos sobre quão psicologicamente adequadas se encontram suas vidas.

Com relação ao domínio social, era esperado que, no meio rural, os idosos apresentassem melhores índices de qualidade de vida subjetiva, pois existe uma maior estabilidade populacional e um sentido de identidade entre as pessoas, o que favorece a manutenção de laços afetivos, maior contato e maior rede de vizinhança, reforçando a interação social (SEQUEIRA; SILVA, 2002). No ambiente urbano, como é menor a quantidade de laços afetivos entre as pessoas e a rede de vizinhos é menor ou inexistente, pode haver uma tendência para o isolamento social, com a chegada da aposentadoria e a perda dos amigos. Porém esta hipótese não foi confirmada neste estudo.

É provável que tanto os participantes urbanos quanto os das cidades rurais, por sua própria idade, já superaram os momentos de grandes mudanças pessoais. Os filhos já estão criados e mantêm seus laços afetivos estabelecidos. Todos são residentes em casas e não em apartamentos e tendem a ficar por bastante tempo na mesma moradia, mantendo relações estáveis com seus vizinhos e amigos e usufruindo das identidades construídas ao longo desse período. Por isso, ainda conservam os benefícios sociais que este tipo de moradia favorece, posto que as pessoas tendem a viver em seu entorno, criando um mundo geográfico próprio. É possível que amostras constituídas por residentes em 
apartamentos ou em megacidades possam apresentar resultados diferentes dos encontrados aqui.

O domínio meio ambiente refere-se a aspectos como segurança física e proteção, cuidados com a saúde e sociais (disponibilidade e qualidade), oportunidades de adquirir novas informações e habilidades, participação e oportunidades de recreação/lazer, entre outros. Sendo assim, a semelhança estatística entre os índices dos idosos dos dois ambientes explica-se pelo fato de que, diferentemente do que ocorria há algumas décadas, as pessoas que vivem no meio rural têm mais acesso à informação, dispõem do atendimento de programas de saúde pública na cidade em que residem e contam com mais opções de lazer, após a chegada da energia elétrica (como televisão, rádio, etc), aproximandose, assim, das características urbanas.

Referindo-se globalmente à qualidade de vida subjetiva, no mesmo ambiente, homens e mulheres não diferiram com relação aos seus índices. O fato de viverem sob condições de qualidade de vida objetiva semelhantes e estarem sujeitos às mesmas limitações promovidas pela idade, justificam a falta de variação nesses índices, em função do gênero.

A fim de verificar a influência da condição econômica sobre este construto, foram comparados os índices de QVS dos idosos que apresentaram renda familiar baixa $(\mathrm{M}=3,36 ; \mathrm{DP}=0,42)$, média $(\mathrm{M}=3,50$; $\mathrm{DP}=0,42)$ e alta $(\mathrm{M}=3,70 ; \mathrm{DP}=0,37)$. De acordo com os resultados encontrados, os índices médios de QVS aumentam conforme aumenta o nível econômico dos idosos $[\mathrm{F}(2,281)=10,97 ; z \leq 0,000]$. Ou seja, os idosos que apresentaram maiores índices de QVS são os que possuem maior renda familiar, assim como os piores índices pertencem aos idosos de renda familiar mais baixa.

Estudos de Trentini (2004) confirmam estes resultados, tendo os idosos de nível econômico mais baixo apresentado os piores índices de qualidade de vida em todos os domínios estudados. Neri (1993) denominou de "efeito tampão", a influência compensatória dos recursos financeiros sobre as perdas da velhice. Estes recursos possibilitam maior acesso à saúde e ao lazer e proporcionam a sensação de auto-eficácia. Ou seja, apesar das perdas e desgastes que acompanham a velhice, o dinheiro possibilita o acesso a serviços de saúde de melhor qualidade, a atividades de lazer e à alimentação adequada, caracterizando melhores 
condições de qualidade de vida objetiva (QVO). E, principalmente, garante ao idoso uma segurança financeira, tornando-se uma preocupação a menos em sua vida e proporcionando uma avaliação mais positiva de sua QVS.

O fato do idoso ser o provedor da renda familiar tem um efeito especial sobre a avaliação da sua QVS. No estudo de Albuquerque, Lobo e Raymundo (1999), realizado no ambiente rural da Paraíba, a aposentadoria ou pensão do idoso, que muitas vezes era a única fonte de renda fixa da família, proporcionava-lhe um importante papel diante da família e da comunidade, além de lhe garantir crédito no comércio local, influenciando tanto na sua qualidade de vida como na sua autoestima.

Foram também comparados os índices de QVS dos idosos de renda baixa do meio rural com os idosos de renda baixa do meio urbano, assim como os índices dos idosos de renda média do rural com os dos idosos de renda média do urbano. Não foram encontradas diferenças significativas entre os grupos, ou seja, com relação à influência da condição econômica, os idosos dos ambientes rural e urbano apresentaram médias estatisticamente semelhantes. Os benefícios que o dinheiro traz, assim como as restrições e preocupações provocadas por sua falta, atingem os idosos de modo semelhante e influenciam a avaliação da QVS independentemente do ambiente em que vivem.

Os idosos de nível econômico alto (com renda familiar maior que R\$3.500,00) não passaram por esta análise, pois no meio rural não foi atingido o número mínimo de participantes necessário para a realização do procedimento estatístico utilizado (Test-t Student), tendo sido encontrados apenas cinco idosos que apresentaram renda familiar alta.

A ausência de pessoas com renda alta ocorre porque, no meio rural, quando as pessoas possuem uma condição financeira mais eleva$\mathrm{da}$, mudam-se para as cidades maiores, mais desenvolvidas, em busca de escolas de melhor qualidade para os filhos, de maior diversidade de entretenimento e lazer para a família, além das expectativas de relacionar-se com pessoas de mesmo nível social. 


\section{Conclusão}

Na maioria das vezes, os estudos acadêmicos se pautam por buscar as diferenças entre os fenômenos estudados. Também este estudo partiu deste pressuposto, pois eram esperados resultados diferenciadores entre os níveis estudados, no que se refere à renda familiar, ao ambiente (urbano ou rural) e ao gênero. Como demonstram os resultados, apenas a renda apresenta níveis comparativos distintos entre os idosos, com os mais favorecidos economicamente mostrando melhores índices de satisfação com a vida. A falta de diferenciação nos índices de QVS, no que diz respeito ao ambiente (urbano ou rural) e ao gênero, aponta para um aspecto importante no processo de desenvolvimento do país.

Parece que, mantendo os mesmos níveis de renda, tanto os homens quanto as mulheres idosas, habitando em cidades grandes ou pequenas, apresentam uma percepção satisfatória sobre a sua qualidade de vida subjetiva. Isto leva a pensar que, superando-se o gargalo da distribuição de renda, as pessoas podem se sentir bem com a vida, tanto nos grandes centros quanto vivendo em pequenas cidades do interior do país. É bom lembrar que, há pouco mais de 30 anos, o Brasil deixou de ter metade de sua população vivendo em ambiente rural, predominantemente em sítios e fazendas, conforme define o IBGE. Atualmente, nota-se que o processo de modernização dos acessos aos serviços tem chegado às pequenas cidades, propiciando melhoria substancial na qualidade de vida dessas pessoas, que sempre foram as menos privilegiadas quanto aos benefícios do estado brasileiro.

Entretanto, deve-se chamar a atenção para o fato de que estes dados dizem respeito aos habitantes de cidades do interior, com populações em torno de 25.000 habitantes. Novos estudos se fazem necessários, ampliando a amostra, para analisar os casos daqueles residentes nos ambientes agrários, sítios, roças e fazendas. Dessa maneira, poderão certamente contribuir para que o poder público possa cumprir seu papel de propiciar as condições adequadas de cidadania para todos os brasileiros, principalmente para aqueles moram nos lugares mais distantes do poder. Esta é uma contribuição que os pesquisadores podem dar a nosso povo, nosso país. 


\title{
EVALUATE OF THE SUBJECTIVE LIFE QUALITY OF AGED: Comparison between residents in agricultural and urban environments.
}

\begin{abstract}
This study has as objective to evaluate the Subjective Life Quality (QVS) of aged residents in urban and agricultural environment of the Paraíba state, in Brazil. They had participated 284 aged ones, 56\% inhabitants of urban environment and $44 \%$ inhabitants of agricultural environment. It was used as instruments the WHOQOL-brief scale and a biological and demographic Questionnaire for characterization of the sample. The results suggest that, in general way, the aged ones possess a satisfactory index of QVS $(\mathrm{M}=3,47 ; \mathrm{DP}=0,43)$. Comparing the groups in function of the sort and the environment where they live it does not have significant difference in the QVS indices; however these indices are correlated directly and significantly with the economic level. It can be concluded that the conditions of life of the urban and agricultural environment, despite the differences, do not influence in the QVS of the aged ones. It makes to reduce the satisfaction with its life conditions is the lack of financial resources, being the groups of minor purchasing power the ones that had demonstrated the lesser indices of QVS.
\end{abstract}

Keywords: Life quality. Aged ones. Agricultural and urban environments.

\section{REFERÊNCIAS}

ALBUQUERQUE, Francisco José Batista; LOBO, Alexandre Lucena; RAYMUNDO, Jorge da Silva. Análise das Repercussões Psicossociais Decorrentes da Concessão de Benefícios Rurais. Psicologia: Reflexão e Crítica, Porto Alegre, v.12, n.2, p.503-520, 1999.

BRASIL. Ministério da Saúde. Conselho Nacional de Saúde. Resolução no 196, de 10 de outubro de 1996. Estabelece as normas de pesquisa envolvendo seres humanos. Bioética, Brasília, DF, v. 4, n. 2, p. 15-25, 1996. Suplemento. 
FLECK, Marcelo Pio de Almeida et al. Aplicação da Versão em Português do Instrumento Abreviado de Avaliação da Qualidade de Vida "WHOQOL-bref". Revista de Saúde Pública, São Paulo, v. 34, n. 2, p. 178-183, 2000.

GOUVEIA, Charlene Nayana Nunes Alves. Avaliação da Qualidade de Vida e do Bem-Estar Subjetivo em Idosos: uma comparação entre os ambiente rural e urbano da Paraíba. João Pessoa: UFPB, 2006.

IBGE. Censo Demográfico. [S. 1.: s. n., 2000]. Disponível em:

$<$ http://www.ibge.gov.br/home/estatistica/populacao/default_censo_2000. shtm>. Acesso em: 26 ago. 2007.

MINAYO, Maria Cecília de Souza; HART, Zulmira Maria de Araújo; BUSS, Paulo Marchiori. Qualidade de vida e saúde: um debate necessário. Ciência e Saúde Coletiva, Rio de janeiro, v. 5, n. 1, p. 7-18, 2000.

NERI, Anita Liberalesso. Qualidade de Vida e Idade Madura. Campinas: Papirus, 1993.

Qualidade de Vida na Velhice e Atendimento Domiciliário. In: DUARTE, Y. A. O.; DIOGO, M. J. D. Atendimento Domiciliar: um enfoque gerontológico. São Paulo: Editora Atheneu, 2000. p. 33-47.

Qualidade de Vida na Velhice. In: REBELATTO, J. R.; MORELLI, J. G. et al. Fisioterapia Geriátrica: a prática da assistência ao idoso. Barueri: Manole, 2004. p. 1-36.

PASCHOAL, Sérgio Márcio Pacheco. Qualidade de Vida do Idoso: elaboração de um instrumento que privilegia sua opinião. 2000. 263f. Dissertação (Mestrado em Medicina)- Departamento de Medicina Preventiva, Faculdade de Medicina, Universidade de São Paulo, 2000. Disponível em: <http://medicina.fm.usp. br/departamento/mpr/info_pos.html >. Acesso em: 23 ago. 2007.

SANTOS, Sérgio Ribeiro et al. Qualidade de Vida do Idoso na Comunidade: aplicação da Escala de Flanagan. Revista Latino-Americana Enfermagem, Ribeirão Preto, v. 10, n. 6, p. 757-764, 2002. 
SEQUEIRA, Arménio; SILVA, Marlene Nunes. O Bem Estar da Pessoa Idosa no Meio Rural. Análise Psicológica, Lisboa, v. 3, p. 505-516, 2002.

SOUSA, Liliana; GALANTE, Helena; FIGUEIREDO, Daniela. Qualidade de Vida e Bem-Estar dos Idosos: um estudo exploratório na população portuguesa. Revista de Saúde Pública, São Paulo, v. 37, n. 3, p. 364-371, 2003.

TRENTINI, Clarissa Marceli. Qualidade de Vida em Idosos: a construção de uma escala de qualidade de vida para idosos - OMS. 2004. 224f. Tese (Doutorado em Psiquiatria) $\neg$ Programa de Pós-Graduação em Ciências Médicas: Psiquiatria, Faculdade de Medicina, Universidade Federal do Rio Grande do Sul, Porto Alegre, 2004.

Recebido em: 06/03/2007

$1^{a}$ revisão: 06/07/2007

$2^{a}$ revisão: $11 / 09 / 2007$

Aceite final: 14/09/2007 